PROCEEDINGS OF THE

AMERICAN MATHEMATICAL SOCIETY

Volume 135, Number 6, June 2007, Pages 1795-1801

S 0002-9939(06)08669-2

Article electronically published on December 28, 2006

\title{
INFINITE DIMENSIONAL UNIVERSAL SUBSPACES GENERATED BY BLASCHKE PRODUCTS
}

\author{
RAYMOND MORTINI
}

(Communicated by Joseph A. Ball)

\begin{abstract}
Let $H^{\infty}$ be the Banach algebra of all bounded analytic functions in the unit disk $\mathbb{D}$. A function $f \in H^{\infty}$ is said to be universal with respect to the sequence $\left(\frac{z+z_{n}}{1+\bar{z}_{n} z}\right)_{n}$ of noneuclidian translates, if the set $\left\{f\left(\frac{z+z_{n}}{1+\bar{z}_{n} z}\right): n \in \mathbb{N}\right\}$ is locally uniformly dense in the set of all holomorphic functions bounded by $\|f\|_{\infty}$. We show that for any sequence of points $\left(z_{n}\right)$ in $\mathbb{D}$ tending to the boundary there exists a closed subspace of $H^{\infty}$, topologically generated by Blaschke products, and linear isometric to $\ell^{1}$, such that all of its elements $f$ are universal with respect to noneuclidian translates. The proof is based on certain interpolation problems in the corona of $H^{\infty}$. Results on cyclicity of composition operators in $H^{2}$ are deduced.
\end{abstract}

During the last two decades problems on cyclicity of operators and universality of functions have gained more and more interest. The achievements are best described in the survey papers [5] and [6] of Grosse-Erdmann. In this paper we study universal Blaschke products, continuing research begun by Heins [7] and complementing known results 2, 1 and 4] on the existence of cyclic vectors for the parabolic and hyperbolic composition operators on the Hardy space $H^{2}$. A first extension of Heins' result was given in [4, where it was shown that for every sequence $\left(z_{n}\right)$ tending to the boundary of the unit disk $\mathbb{D}=\{z \in \mathbb{C}:|z|<1\}$ there exists a Blaschke product $B$ universal for noneuclidian translates, meaning that the set $\left\{B\left(\left(z+z_{n}\right) /\left(1+\overline{z_{n}} z\right)\right): n \in \mathbb{N}\right\}$ is locally uniformly dense in the set $\mathscr{B}$ of all analytic functions in $\mathbb{D}$ bounded by one. This gave rise to a Blaschke product that was a joint cyclic vector for a given sequence of composition operators $\mathcal{C}_{\varphi_{n}}$ on $H^{2}$. Recall that a vector $x$ is called cyclic for an operator $T$ defined on a Fréchet space $X$, if the linear span of the orbit $\left\{T^{n} x: x \in X\right\}$ is dense in $X$. Here the symbols $\varphi_{n}$ were chosen from the set of hyperbolic and parabolic automorphisms of the unit disk. In another direction, Aron and Gorkin [1] showed that in the Banach algebra $H^{\infty}\left(\mathbb{B}_{n}\right)$ of the ball $\mathbb{B}_{n}$ in $\mathbb{C}^{n}(n \geq 1)$, there exists a closed, infinite-dimensional subspace such that all its norm one elements are universal with respect to some sequence of automorphisms. For the reader's convenience, we shall define an extended concept of universal elements.

Received by the editors September 6, 2005 and, in revised form, February 5, 2006.

2000 Mathematics Subject Classification. Primary 30D50; Secondary 47B33, 46J15, 30H05.

Key words and phrases. Universal Blaschke products, interpolation in the corona, composition operators on Hardy spaces, joint cyclic vectors.

The author thanks the referee for his/her comments improving the exposition of this work.

(C)2006 American Mathematical Society Reverts to public domain 28 years from publication 1795 
Definition. Let $\left(z_{n}\right)$ be a sequence in $\mathbb{D}$ converging to the boundary, $\partial \mathbb{D}$, of $\mathbb{D}$. A function $f \in H^{\infty}$ is said to be universal (with respect to the sequence $\left(\frac{z+z_{n}}{1+\bar{z}_{n} z}\right)$ of noneuclidian translates, or more briefly with respect to $\left.\left(z_{n}\right)\right)$ if the set $\left\{f\left(\frac{z+z_{n}}{1+\bar{z}_{n} z}\right)\right.$ : $n \in \mathbb{N}\}$ is locally uniformly dense in the set of all holomorphic functions bounded by $\|f\|_{\infty}$.

We note that a function $f \not \equiv 0$ is universal in our new sense if and only if $f /\|f\|_{\infty}$ is universal in the sense of Heins. We are now concerned with the following question: Are there any closed, infinite-dimensional subspaces of $H^{\infty}$, topologically generated by Blaschke products, such that all of its elements are universal with respect to some given sequence $\left(z_{n}\right)$ in $\mathbb{D}$ ? In this paper we shall be able to construct such a subspace with the additional property that it is linear isometric to $\ell^{1}$. We also find uniformly closed subspaces in $H^{2}$, all of whose nonzero elements are cyclic vectors for a given (sequence) of hyperbolic/parabolic composition operators.

Our methods are based on solving appropriate interpolation problems on the corona of $H^{\infty}$. It is assumed that the reader is familiar with Hoffman's theory [8] on the structure of the spectrum (or maximal ideal space) of $H^{\infty}$. A good reference is also Garnett's book [3]. So let $M\left(H^{\infty}\right)$ be the the space of nonzero multiplicative linear functionals on $H^{\infty}$ endowed with the weak-* topology. By Carleson's Corona Theorem, we may view $M\left(H^{\infty}\right)$ as a compactification of the unit disk so that every function $f \in H^{\infty}$ has a (unique) continuous extension to $M\left(H^{\infty}\right)$. This extension is given by the Gelfand transform $\hat{f}$ defined as $\hat{f}(m):=m(f)$ whenever $m \in M\left(H^{\infty}\right)$. As usual we shall identify $\hat{f}$ with $f$. The corona of $H^{\infty}$ is defined to be $M\left(H^{\infty}\right) \backslash \mathbb{D}$. It is customary to identify this set with the maximal ideal space $M\left(H^{\infty}+C\right)$ of the algebra $H^{\infty}+C$ of sums of boundary values of bounded analytic functions and complex-valued continuous functions on the unit circle $T=\{z \in \mathbb{D}:|z|=1\}$.

Let $m \in M\left(H^{\infty}\right) \backslash \mathbb{D}$ and let $\left(z_{n}\right)$ be a sequence in $\mathbb{D}$ capturing $m$ in its closure; say $z_{n(\alpha)} \rightarrow m$ for some subnet $\left(z_{n(\alpha)}\right)$ of $\left(z_{n}\right)$. According to Hoffman's theory the map $L_{m}: \mathbb{D} \rightarrow M\left(H^{\infty}\right)$ given by

$$
L_{m}(z)=\lim \frac{z+z_{n(\alpha)}}{1+\overline{z_{n(\alpha)}} z}
$$

where the limit is taken in the product-topology for $M\left(H^{\infty}\right)^{\mathbb{D}}$, is well defined, satisfies $L_{m}(0)=m$ and has the property that for each $f \in H^{\infty}$

$$
\left(\hat{f} \circ L_{m}\right)(z)=\lim f\left(\frac{z+z_{n(\alpha)}}{1+\overline{z_{n(\alpha)}} z}\right)
$$

is a function in $H^{\infty}$ again.

These maps, which are seen to be limits of certain hyperbolic automorphisms, allow us to relate problems on universality to interpolation problems in $M\left(H^{\infty}\right)$. For additional background information, we also refer the reader to 4 .

To conclude this introduction, let us recall some notation. A thin Blaschke product is a function of the form

$$
b(z)=\prod_{n=1}^{\infty} \frac{\bar{a}_{n}}{\left|a_{n}\right|} \frac{a_{n}-z}{1-\bar{a}_{n} z}
$$


where the $\left(a_{n}\right)$ satisfy the condition

$$
\lim _{k \rightarrow \infty} \prod_{n: n \neq k}\left|\frac{a_{n}-a_{k}}{1-\bar{a}_{n} a_{k}}\right|=1 .
$$

Note that this condition is equivalent to $\left(1-\left|a_{n}\right|^{2}\right)\left|b^{\prime}\left(a_{n}\right)\right| \rightarrow 1$. Properties of this kind of Blaschke product that are relevant to the present paper, were derived in 4]. Finally, for a function $f \in H^{\infty}$, we define its zero set on the corona of $H^{\infty}$ by $Z(f)=\left\{x \in M\left(H^{\infty}\right) \backslash \mathbb{D}: f(x)=0\right\}$.

\section{JOINT INTERPOLATION OF FUNCTIONS}

For better reference, we point out the following facts, well known to specialists in this area (see e.g. [3]). Recall that an interpolating sequence $\left(x_{n}\right)$ for a uniform algebra $A$ is a sequence of points in the spectrum of $A$ such that for every $\left(w_{n}\right) \in \ell^{\infty}$ there exists $f \in A$ with $f\left(x_{n}\right)=w_{n}$ for all $n$.

Lemma 1.1. Let $\left(m_{\alpha}\right)$ be a net in $M\left(H^{\infty}+C\right)$ converging to $m$. Then $L_{m_{\alpha}}$ converges to $L_{m}$, i.e. $f \circ L_{m_{\alpha}}$ converges locally uniformly to $f \circ L_{m}$ for every $f \in H^{\infty}$.

A set or sequence $\left(x_{n}\right)$ of distinct points in a compact Hausdorff space is called discrete, if for every $n$ there exists an open neighborhood $U_{n}$ of $x_{n}$ such that $U_{n}$ does not contain any other of the points $x_{k}, k \neq n$.

Lemma 1.2. (a) Let $b$ be an interpolating Blaschke product. Then $Z(b)$ is homeomorphic to $\beta \mathbb{N} \backslash \mathbb{N}$, where $\beta \mathbb{N}$ is the Stone-Cech compactification of $\mathbb{N}$.

(b) Let $\left(x_{n}\right)$ be a discrete subsequence of $\beta \mathbb{N} \backslash \mathbb{N}$. Then $\left(x_{n}\right)$ is an interpolating sequence for $C(\beta \mathbb{N})$.

(c) If $b$ is an interpolating Blaschke product, then any discrete sequence of points $x_{n} \in Z(b)$ is an interpolating sequence for $H^{\infty}$ and the closure of $\left\{x_{n}: n \in \mathbb{N}\right\}$ is homeomorphic to $\beta \mathbb{N}$.

(d) Any sequence of distinct points in $Z(b)$ admits a discrete subsequence.

The main tool in this section will be the following interpolational result from ([4], p. 179). Recall that $\mathscr{B}$ denotes the set of all functions in $H^{\infty}$ bounded by one.

Theorem A. Let $b$ be a thin Blaschke product and let $\left(m_{n}\right)$ be a discrete sequence of points in $Z(b)$. Then for any sequence $\left(c_{n}\right)$ of finite Blaschke products there exists a Blaschke product $B$ such that $B \circ L_{m_{n}}=c_{n}$ for every $n$.

It was shown in 4 that such a function $B$ is universal for the zero sequence of $b$ in $\mathbb{D}$, whenever $\left\{c_{n}: n \in \mathbb{N}\right\}$ is dense in $\mathscr{B}$, which provided the link between interpolation and universality. To construct vector spaces consisting of universal functions we need the following extensions. At this point I want to thank Artur Nicolau who, in 2001, wondered whether there exist Blaschke products with the property given in the following proposition.

Proposition 1.3. There exists a pair of Blaschke products $\left(B_{1}, B_{2}\right)$ such that for every $\left(f_{1}, f_{2}\right) \in \mathscr{B} \times \mathscr{B}$ there are uncountably many $m \in M\left(H^{\infty}+C\right)$ such that $B_{j} \circ L_{m}=f_{j}$ for $j=1,2$.

Proof. Let $b$ be a thin Blaschke product. Choose a double indexed, discrete subset $\left\{x_{j, k}:(j, k) \in \mathbb{N}^{2}\right\}$ of points in $Z(b)$. According to Carathéodory's approximation 
theorem ([3], p. 6), let $\left\{q_{k}: k \in \mathbb{N}\right\}$ and $\left\{p_{j}: j \in \mathbb{N}\right\}$ be two countable sets of finite Blaschke products that are locally uniformly dense in $\mathscr{B}$. By Theorem A there are Blaschke products $B_{1}$ and $B_{2}$ such that

$$
B_{2} \circ L_{x_{j, k}}=q_{k} \text { and } B_{1} \circ L_{x_{j, k}}=p_{j} \text { for every } j, k \in \mathbb{N} \text {. }
$$

Let $E_{j}=\overline{\left\{x_{j, k}: k \in \mathbb{N}\right\}} \backslash\left\{x_{j, k}: k \in \mathbb{N}\right\}$. Then $B_{1} \circ L_{x_{j}}=p_{j}$ for every $x_{j} \in E_{j}$ (note that if $x_{\alpha} \rightarrow x$, then $L_{x_{\alpha}} \rightarrow L_{x}$ ).

Let $\left(f_{1}, f_{2}\right) \in \mathscr{B} \times \mathscr{B}$. Choose a translation-invariant distance function $d$ on $H(\mathbb{D})$, the space of all holomorphic functions on $\mathbb{D}$, which generates local uniform convergence. Let $q_{k_{n}}$ be a subsequence of $\left(q_{k}\right)$ with $d\left(q_{k_{n}}, f_{2}\right) \rightarrow 0$. Choose $x_{j} \in$ $\overline{\left\{x_{j, k_{n}}: n \in \mathbb{N}\right\}} \backslash\left\{x_{j, k}: k \in \mathbb{N}\right\}$. Then $x_{j} \in E_{j}$ and $B_{2} \circ L_{x_{j}}=f_{2}$.

Let $p_{j_{l}}$ be a subsequence of $\left(p_{j}\right)$ with $d\left(p_{j_{l}}, f_{1}\right) \rightarrow 0$. Note that $B_{1} \circ L_{x_{j_{l}}}=p_{j_{l}}$ for every $l$. Let $m$ be a cluster point of the $x_{j_{l}}$. Then $B_{2} \circ L_{m}=f_{2}$ and $B_{1} \circ L_{m}=f_{1}$. Since by Lemma 1.2 each discrete subsequence of $\left(x_{j_{l}}\right) \in Z(b)^{\mathbb{N}}$ is an interpolating sequence, hence homeomorphic to the Stone-Čech compactification of $\mathbb{N}$, we see that for every pair $\left(f_{1}, f_{2}\right) \in \mathscr{B} \times \mathscr{B}$ there are uncountably many $m \in Z(b)$ such that $B_{1} \circ L_{m}=f_{1}$ and $B_{2} \circ L_{m}=f_{2}$.

In our next theorem we generalize this interpolation result for pairs of Blaschke products to sequences.

Theorem 1.4. There exists a sequence of Blaschke products $\left(B_{1}, B_{2}, \ldots\right)$ such that for every $\left(f_{1}, f_{2}, \ldots\right) \in \mathscr{B}^{\mathbb{N}}$ there are uncountably many $m \in M\left(H^{\infty}+C\right)$ such that $B_{j} \circ L_{m}=f_{j}$ for every $j \in \mathbb{N}$.

Proof. The proof will be done by induction. The case $n=1$ and $n=2$ are done in Proposition 1.3. To proceed, we assume that an $n$-tuple of Blaschke products $\left(B_{1}, \cdots, B_{n}\right)$ has been constructed with the property that for every $\left(f_{1}, \cdots, f_{n}\right) \in$ $\mathscr{B}^{n}$ there exist uncountably many $m \in Z(b)$ such that $B_{j} \circ L_{m}=f_{j}$ for $j=1, \cdots, n$. Let $\mathscr{P}=\left\{p_{k}: k \in \mathbb{N}\right\}$ and $\left\{q_{k}: k \in \mathbb{N}\right\}$ be two countable, dense subsets of finite Blaschke products. We fix an enumeration of the countable set of indices $\left\{\left(k_{1}, \cdots, k_{n}\right): k_{s} \in \mathbb{N}\right\}$, say $\omega_{j}, j=1,2 \cdots$. For $\omega \in \mathbb{N}^{n}$, let $\mathbf{p}_{\omega} \in \mathscr{P}^{n}$. Decompose $b$ into the product of infinitely many (infinite) thin Blaschke products $b_{j}$ (see e.g. [4, section 1). Choose for every $\omega_{j}$ an infinite number of points $m \in Z\left(b_{j}\right)$ such that $\left(B_{1}, \cdots, B_{n}\right) \circ L_{m}=\mathbf{p}_{\omega_{j}}$. Enumerate these points as $x_{\omega_{j}, k}, k \in \mathbb{N}$. By Lemma 1.2(d), we may assume that for any $j,\left\{x_{\omega_{j}, k}: k \in \mathbb{N}\right\}$ is a discrete subset of $Z\left(b_{j}\right)$. Since $Z\left(b_{j}\right) \cap \overline{\bigcup_{k \neq j} Z\left(b_{k}\right)}=\emptyset$, the whole set $\left\{x_{\omega_{j}, k}: j, k \in \mathbb{N}\right\}$ is discrete. Hence, by Theorem A, there exists a Blaschke product $B_{n+1}$ such that $B_{n+1} \circ L_{x_{\omega_{j}, k}}=q_{k}$ for every $j$ and $k \in \mathbb{N}$. As in the proof of Proposition 1.3 we see that whenever $f_{n+1} \in \mathscr{B}$ is approached by some sequence $\left(q_{k_{\nu}}\right)_{\nu}$, and $\left(f_{1}, \cdots, f_{n}\right)$ by a sequence $\left(\mathbf{p}_{\omega_{j_{l}}}\right)_{l}$, then for every cluster point $m$ of $x_{j_{l}}, x_{j_{l}} \in \overline{\left\{x_{\omega_{j_{l}}, k_{\nu}}: \nu \in \mathbb{N}\right\}} \backslash\left\{x_{\omega_{j}, k}: k \in \mathbb{N}\right\}$, we have $\left(B_{1}, \cdots, B_{n}, B_{n+1}\right) \circ L_{m}=\left(f_{1}, \cdots, f_{n}, f_{n+1}\right)$.

This ends the construction of the $B_{n}$. Now fix $\left(f_{1}, f_{2}, \ldots\right) \in \mathscr{B}^{\mathbb{N}}$. For each $n \in \mathbb{N}$, take $m_{n} \in M\left(H^{\infty}+C\right)$ so that $\left(B_{1}, \cdots, B_{n}\right) \circ L_{m_{n}}=\left(f_{1}, \cdots, f_{n}\right)$. Let $m$ be any cluster point of these $m_{n}$, say $m=\lim m_{n(\alpha)}$ for some subnet $\left(m_{n(\alpha)}\right)$ of $\left(m_{n}\right)$. Fix $N \in \mathbb{N}$. Choose $\alpha$ so big that $n(\alpha)>N$. Then, by Lemma 1.1 . $B_{N} \circ L_{m}=\lim B_{N} \circ L_{m_{n(\alpha)}}=f_{N}$.

As a by-product, we obtain the following interesting observation. 
Corollary 1.5. There exists a closed subspace $M$ in $H^{\infty}$ that is linear isometric to $\ell^{1}$.

Proof. Let $M$ be the supnorm-closure of the vector space $V$ generated by the Blaschke products $B_{n}$ above. Let $f=\sum_{j=1}^{N} \lambda_{j} B_{j} \in V$. We claim that

$$
\|f\|_{\infty}=\sum_{j=1}^{N}\left|\lambda_{j}\right| \text {. }
$$

In fact, choose according to Theorem $1.4 m \in M\left(H^{\infty}+C\right)$ so that $B_{j}(m)=$ $\exp \left(-i \arg \lambda_{j}\right)$ for all $j \in\{1, \ldots, N\}$. Then

$$
\sum_{j=1}^{N}\left|\lambda_{j}\right| \geq|| f \|_{\infty} \geq|f(m)|=\left|\sum_{j=1}^{N} \lambda_{j} B_{j}(m)\right|=\sum_{j=1}^{N}\left|\lambda_{j}\right| .
$$

This proves (1.1). The same norm equality also holds whenever $f=\sum_{j=1}^{\infty} \lambda_{j} B_{j}$, where $\left(\lambda_{j}\right) \in \ell^{1}$. Since $\left\{\sum_{j=1}^{\infty} \lambda_{j} B_{j}:\left(\lambda_{j}\right) \in \ell^{1}\right\} \subseteq M$, we obtain a linear isometry of $\ell^{1}$ into $M$. But the image of $\ell_{1}$ under the isometry $\left(\lambda_{j}\right) \mapsto \sum_{j=1}^{\infty} \lambda_{j} B_{j}$ is supnormcomplete; hence this map is onto and $M=\left\{\sum_{j=1}^{\infty} \lambda_{j} B_{j}:\left(\lambda_{j}\right) \in \ell^{1}\right\}$.

\section{UNIVERSAL SUBSPACES}

The following result is a very useful characterization of universality.

Proposition 2.1. A function $f \in H^{\infty}$ is universal (for a sequence $\left(z_{n}\right)$ ) if and only if for every $g \in H^{\infty}$ with $\|g\|_{\infty} \leq\|f\|_{\infty}$ there exists a point $m \in \overline{\left\{z_{n}: n \in \mathbb{N}\right\}}$ such that $f \circ L_{m}=g$.

Proof. Suppose that $f$ is universal. Let $\|g\|_{\infty} \leq\|f\|_{\infty}$. There is a subsequence $\left(z_{n_{k}}\right)$ such that $f\left(\left(z+z_{n_{k}}\right) /\left(1+\bar{z}_{n_{k}} z\right)\right)$ tends locally uniformly in $\mathbb{D}$ to $g$. Let $m \in M\left(H^{\infty}+C\right)$ be a cluster point of $\left(z_{n_{k}}\right)$. Then a subnet $\left(z_{n_{k(\alpha)}}\right)$ converges to $m$. By Lemma 1.1, for every $z \in \mathbb{D}$,

$$
\left(f \circ L_{m}\right)(z)=\lim f\left(\left(z+z_{n_{k(\alpha)}}\right) /\left(1+\bar{z}_{n_{k(\alpha)}} z\right)\right)=g(z) .
$$

On the other hand, if for some $m \in \overline{\left\{z_{n}: n \in \mathbb{N}\right\}}$ we have $f \circ L_{m}=g$, then of course $\|g\|_{\infty} \leq\|f\|_{\infty}$. By Hoffman's theory, $L_{m}=\lim L_{z_{n(\alpha)}}$ for some subnet $\left(z_{n(\alpha)}\right)$ of $\left(z_{n}\right)$ and so $g(z)=\left(f \circ L_{m}\right)(z)=\lim f\left(\left(z+z_{n(\alpha)}\right) /\left(1+\bar{z}_{n(\alpha)} z\right)\right)$. It follows that there exists a subsequence $\left(z_{n_{k}}\right)$ such that $f\left(\left(z+z_{n_{k}}\right) /\left(1+\bar{z}_{n_{k}} z\right)\right)$ tends to $g(z)$.

Proposition 2.2. Let $M \subseteq H^{\infty}$ be a set all of whose elements are universal (with respect to a sequence $\left(z_{n}\right)$.) Then every element in the uniform closure of $M$ is universal, too.

Proof. Let $\left(f_{n}\right)$ be a sequence of universal elements in $M$ that converges uniformly to some $f \in H^{\infty}$. We may assume that $f \not \equiv 0$, since the zero function is universal in view of our definition.

Let $g \in H^{\infty}$ such that $\|g\|_{\infty} \leq\|f\|_{\infty}$. Since $f_{n}$ is universal and $\left\|\frac{\left\|f_{n}\right\|_{\infty}}{\|f\|_{\infty}} g\right\|_{\infty} \leq$ $\left\|f_{n}\right\|_{\infty}$, we may choose by Proposition $2.1 m_{n} \in \overline{\left\{z_{j}: j \in \mathbb{N}\right\}}$ such that

$$
f_{n} \circ L_{m_{n}}=\frac{\left\|f_{n}\right\|_{\infty}}{\|f\|_{\infty}} g .
$$


Let $m$ be a cluster point of the $m_{n}$, say $m_{n(\alpha)} \rightarrow m$. The associated subnet $\left(f_{n(\alpha)}\right)$ also converges to $f$. Since the convergence is uniform, we have

$$
\lim \left(f_{n(\alpha)} \circ L_{m_{n(\alpha)}}\right)(z) \rightarrow\left(f \circ L_{m}\right)(z) .
$$

Because $\left\|f_{n}\right\|_{\infty} \rightarrow\|f\|_{\infty}$, we conclude that $f \circ L_{m}=g$. Thus, by Proposition 2.1. $f$ is universal.

We can now state the main theorem of this note.

Theorem 2.3. Let $\left(z_{n}\right)$ be a sequence in $\mathbb{D}$ such that $\left|z_{n}\right| \rightarrow 1$. Then there exists a uniformly closed subspace, $M$, of $H^{\infty}$, topologically generated by Blaschke products and linear isometric to $\ell^{1}$, such that each element $f \in M$ is universal for the sequence $\left(\frac{z+z_{n}}{1+\bar{z}_{n} z}\right)_{n}$ of noneuclidian translates.

Proof. Let $b$ be a thin Blaschke product whose zeros are contained within the set $\left\{z_{n}: n \in \mathbb{N}\right\}$; see e.g. ([4, p. 178). By Theorem 1.4 there exist Blaschke products $B_{j}$ such that for every $\left(f_{1}, f_{2}, \cdots\right) \in \mathscr{B}^{\mathbb{N}}$ there is $m \in Z(b)$ such that for every $j$ one has $B_{j} \circ L_{m}=f_{j}$. Let $V$ be the vector space generated by these $B_{j}$ and let $M$ be the closure of $V$. By Corollary 1.5, $M$ is linear isometric to $\ell^{1}$. Moreover, let $f=\sum_{j=1}^{N} \lambda_{j} B_{j} \in V$. We prove that $f$ is universal. To this end, let $g \in H^{\infty}$ and $\|g\|_{\infty} \leq\|f\|_{\infty}$. Note that by (1.1), $\|f\|_{\infty}=\sum_{j=1}^{N}\left|\lambda_{j}\right|$. In view of the choice of the $B_{j}$, there is $m \in Z(b)$ so that for $j \in\{1, \cdots, N\}$

$$
B_{j} \circ L_{m}=\left[\exp \left(-i \arg \lambda_{j}\right)\right] g /\|f\|_{\infty} .
$$

Thus

$$
f \circ L_{m}=\sum_{j=1}^{N} \lambda_{j} B_{j} \circ L_{m}=\left(g /\|f\|_{\infty}\right) \cdot \sum_{j=1}^{N}\left|\lambda_{j}\right|=\left(g /\|f\|_{\infty}\right)\|f\|_{\infty}=g .
$$

Therefore, by Proposition 2.1, $f$ is universal. Proposition 2.2 now implies that every $f \in \bar{V}=M$ is universal.

\section{CyClicity OF COMPOSITION OPERATORS IN $H^{2}$}

The material in section 2 will allow us to derive a result on the existence of infinite-dimensional subspaces in $H^{2}$ each of whose nonzero elements are cyclic vectors for a countable set of nonelliptic, invertible composition operators.

Theorem 3.1. Let $\left(\varphi_{n}\right)$ be a sequence of hyperbolic or parabolic automorphisms in $\mathbb{D}$. Then there exists a uniformly closed subspace $M$ of $H^{2}$ topologically generated by Blaschke products such that each element $f \in M \backslash\{0\}$ is a joint cyclic vector for the sequence of composition operators $\left\{\mathcal{C}_{\varphi_{n}}: n \in \mathbb{N}\right\}$ on the Hardy space $H^{2}$.

Proof. This assertion can be deduced from Theorem 1.4 along the same lines as the assertion of [4, Theorem 3.2. We just need to represent these automorphisms $\varphi(z)=e^{i \theta} \frac{a-z}{1-\bar{a} z}(-\pi \leq 0<\pi)$ as $\varphi=L_{e^{i \theta} a} \circ R_{\theta+\pi}$, where $R_{\beta}(z)=e^{i \beta} z$ is a rotation with angle $\beta \in\left[0,2 \pi\left[\right.\right.$. The orbits of the iterates $\varphi^{[j]}$ of $\varphi$ at 0 then play the role of the $\left(z_{j}\right)$.

This will yield Blaschke products $B_{k}$ such that every function $f$ in the vector space generated by them in $H^{\infty}$ has the property that $\left\{f \circ \varphi_{n}^{[j]}: j \in \mathbb{N}\right\}$ is locally uniformly dense in $\left\{h \in H^{\infty}:\|h\|_{\infty} \leq\|f\|_{\infty}\right\}$ for every $n$. Hence the linear span of $\left\{\mathcal{C}_{\varphi_{n}}^{j}(f): j \in \mathbb{N}\right\}$ is norm dense in $H^{2}$, whenever $f \not \equiv 0$. 


\section{REFERENCES}

[1] R. Aron, P. Gorkin: An infinite dimensional vector space of universal functions for $H^{\infty}$ on the ball, to appear in Canad. Math. Bull.

[2] P. Bourdon, Shapiro, J. H.: Cyclic phenomena for composition operators. Mem. Amer. Math. Soc. 125 (1997), no. 596, x+105 pp. MR1396955 (97h:47023)

[3] J.B. Garnett: Bounded Analytic Functions, Academic Press, New York, 1981. MR0628971 (83g:30037)

[4] P. Gorkin, R. Mortini: Universal Blaschke products, Math. Proc. Camb. Phil. Soc. 136 (2004), 175-184. MR2034021 (2004m:30056)

[5] K.-G. Grosse-Erdmann: Universal families and hypercyclic operators, Bull. Amer. Math. Soc. 36 (1999), 345-381. MR1685272 (2000c:47001)

[6] K.-G. Grosse-Erdmann: Recent developments in hypercyclicity, RACSAM Rev. R. Acad. Cienc. Exactas Fis. Nat. Ser. A Mat. 97 (2003), 273-208. MR2068180 (2005c:47010)

[7] M. Heins: A universal Blaschke product, Archiv Math. 6 (1955), 41-44. MR0065644 $(16: 460 \mathrm{e})$

[8] K. Hoffman: Bounded analytic functions and Gleason parts. Ann. Math. 86 (1967), 74-111. MR0215102(35:5945)

Département de Mathématiques, Université Paul Verlaine, Ile du Saulcy F-57045 Metz, France

E-mail address: mortini@math.univ-metz.fr 